\title{
Health-Related Quality of Life Measurement Inpatient Geriatric Rehabilitation: A Comparison of the Feasibility and Suitability Between the SF-36 and EQ-5D-5L Questionnaires
}

\author{
Grund $S^{1 *}$, Breitinger $E^{2}$, Fricke $S^{2}$, Alpers $\mathbf{G W}^{3}$, Hundsdorfer $\mathbf{W}^{4}$ and Schäfer HG $^{4}$ \\ ${ }^{1}$ Department of Geriatrics, Diakonissen Hospital Mannheim, Mannheim Germany \\ ${ }^{2}$ Department of Psychology, University of Mannheim, Mannheim, Germany \\ ${ }^{3}$ Department of Psychology and Otto-Selz-Institute, University of Mannheim, Mannheim, Germany \\ ${ }^{4}$ Department of Geriatric Rehabilitation, Diakonissen Hospital Mannheim, Mannheim Germany
}

\begin{abstract}
Background: A key goal of geriatric rehabilitation is the improvement of health-related quality of life (HRQoL). Although recommended in several medical guidelines the measurement of $\mathrm{HRQ}$ and it is not yet examined, whether and how this could be routinely measured in an in-patient geriatric rehabilitation setting.
\end{abstract}

Method: In a prospective cross-sectional study, 86 patients from our rehabilitation clinic completed the SF-36 and the EQ-5D-5L. We analysed the questionnaires' feasibility and suitability operationalized in the needed assistance, the amount of time for completion, occurring questions and missing values.

Results: $46.5 \%$ of the participants needed strong assistance completing the SF-36 with an average completion time of $11: 36 \mathrm{~min}$. Items concerning the physical dimensions showed the most missing values as well as items referring to 'daily activities, at work or at home'. $41.9 \%$ of the participants relied on strong assistance for the completion of the EQ-5D-5L. The item referring to 'usual activities as e.g. work' had the most missing values.

Conclusion: The general feasibility of the SF-36 and EQ-5D-5L for measuring HRQoL in in-patient geriatric rehabilitation could be confirmed. However, the EQ-5D-5L seems to be more suitable for a routinely use. Due to the items, mental health might be underrepresented compared to the SF36.

Keywords: Geriatric rehabilitation; Health-related quality of life; Oldest age

\section{Introduction}

The improvement of health-related quality of life (HRQoL) is the central overriding goal of geriatric rehabilitation [1,2]. Its measurement becomes more important [3] and is recommended in different guidelines [4-6]. However, it is performed neither extensively nor routinely, mainly due to the fact that the most appropriate measurement remains unclear. The SF-36 and the EQ-5D-5L are two questionnaires measuring HRQoL. They are increasingly used worldwide $[7,8]$, both have good validity and reliability $[9,10]$, are suitable for older adults $[11,12]$ and are recommended for rehabilitation procedures [4-6].

\section{Research Questions}

The present study seeks to answer the question whether the SF-36 and EQ-5D-5L questionnaires are feasible and

suitable in an in-patient geriatric rehabilitation setting for routinely use as a self-completion measurement. The time for completion of the SF-36 and EQ-5D-5L questionnaires as well as the extent of required assistance are depictured, in addition by the missing values.

\section{Methodology}

\section{Participants}

Between January 20, 2016 and March 18, 2016, 86 multimorbid geriatric in-patients $(69.8 \%$ women, average age $80.98 \mathrm{y}, \mathrm{SD}=7.4)$ of the Clinic for Geriatric Rehabilitation at the Diakonissenkrankenhaus Mannheim completed the SF-36 and the EQ-5D-5L during the first three days of their stay. The recruitment rate was $58.1 \%$ and the dropout rate $11.3 \%$. The patients' primary diagnosis can be found in Appendix 1 in the supplementary data on the journal website.

\section{Materials}

HRQoL was evaluated using the SF-36 version for self-completion, and the EQ-5D-5L along with its visual analogue scale (VAS) to self-rate health quality. EQ-5D-5L indices were calculated with the 'Crosswalk Index Value Calculator' provided on the EuroQol website [13]. The questionnaires' feasibility and suitability were operationalized in the extent of needed assistance, time for completion, and missing values. The number of questions asked/comments made about single items as an indicator of comprehension problems was documented. Moreover, we compared the results with representative German samples.

\section{Statistical analysis}

The data collection and statistical evaluation were carried out with SPSS (IBM, version 19). The relationship between the EQ-5D-5L and the SF-36 was examined with the Pearson correlation coefficient using twotailed significance testing ( $\alpha=5 \%$ ). Multiple t-tests were calculated for the comparisons between the current sample and the German representative samples ( $\alpha=1 \%$, conservative $\alpha$-level due to multiple testing).

*Corresponding author: Stefan Grund, Department of Geriatrics, Diakonissen Hospital Mannheim, Mannheim Germany, Tel: 004962181023650; E-mail: stefan.grund@diakonissen.de

Received December 21, 2017; Accepted December 28, 2017; Published December 29, 2017

Citation: Grund S, Breitinger E, Fricke S, Alpers GW, Hundsdörfer W, et al. (2017) Health-Related Quality of Life Measurement Inpatient Geriatric Rehabilitation: A Comparison of the Feasibility and Suitability Between the SF-36 and EQ-5D-5L Questionnaires. J Gerontol Geriatr Res 6: 458. doi:10.4172/2167-7182.1000458

Copyright: () 2017 Grund S, et al. This is an open-access article distributed under the terms of the Creative Commons Attribution License, which permits unrestricted use, distribution, and reproduction in any medium, provided the original author and source are credited. 
Citation: Grund S, Breitinger E, Fricke S, Alpers GW, Hundsdörfer W, et al. (2017) Health-Related Quality of Life Measurement Inpatient Geriatric Rehabilitation: A Comparison of the Feasibility and Suitability Between the SF-36 and EQ-5D-5L Questionnaires. J Gerontol Geriatr Res 6: 458. doi:10.4172/2167-7182.1000458

Page 2 of 4

\section{Results}

\section{Feasibility of the SF-36 and EQ-5D-5L}

The amount of time the patients needed for the completion of the SF-36 and EQ-5D-5L is shown in Table 1 as well as the extent of needed assistance. Patients in the category 'no assistance' completed the questionnaires completely on their own. The category 'some assistance' comprises patients who could complete the questionnaires alone but had questions of understanding which were answered by the investigator. All items were read out loud by the investigator to patients in the category 'strong assistance' (Appendix 2 'reasons for strong support' on the website).

\section{Missing values}

Items of the SF-36 dimensions Social Functioning, Role Physical, Role Emotional, and Pain had the most missing values (soc2 $\mathrm{N}=8$; rolph1, rolph4, rolem3 $\mathrm{N}=6$ each; pain2 $\mathrm{N}=7$ ). Furthermore, $50 \%$ $(23 / 46)$ of the participants who self-completed the questionnaire

\begin{tabular}{|c|c|c|c|c|}
\hline SF-36 & No assistance & $\begin{array}{c}\text { Some } \\
\text { assistance }\end{array}$ & $\begin{array}{c}\text { Strong } \\
\text { assistance }\end{array}$ & Total \\
\hline M & $14: 11$ & $13: 07$ & $11: 36$ & $12: 29$ \\
\hline SD & $10: 22$ & $05: 22$ & $03: 45$ & $05: 10$ \\
\hline N & $6(7 \%)$ & $40(46.5 \%)$ & $40(46.5 \%)$ & $86(100 \%)$ \\
\hline \multicolumn{5}{|c|}{ EQ-5D-5L } \\
\hline M & $03: 02$ & $04: 15$ & $03: 06$ & $03: 42$ \\
\hline SD & $01: 45$ & $02: 18$ & $01: 10$ & $01: 57$ \\
\hline N & $5(5.8 \%)$ & $45(52.3 \%)$ & $36(41.9 \%)$ & $86(100 \%)$ \\
\hline M: Mean Value, SD: Standard Deviation, N- Sample Size \\
\hline
\end{tabular}

Table 1: Needed assistance and duration of the completion of the SF-36 and the EQ-5D-5L. The values displayed for means and standard deviations represent time in minutes.

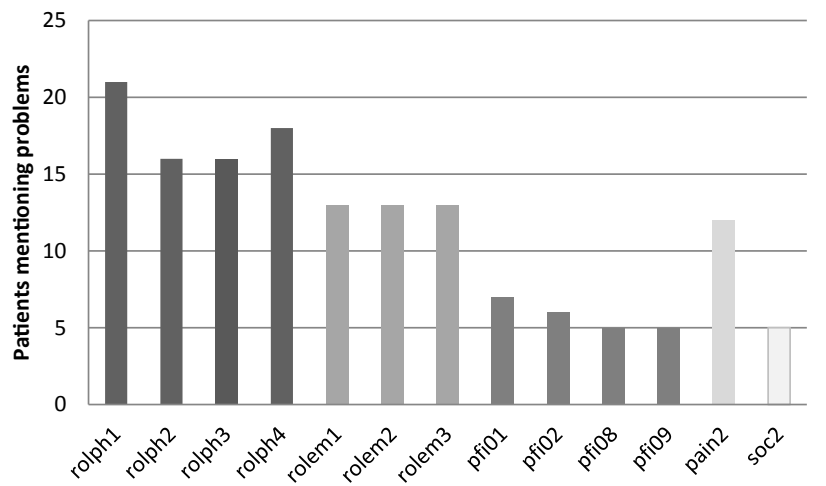

Corresponding dimensions: rolph: Role-Physical, rolem: Role-Emotional, pfi: Physical Funcitioning, pain: Bodily Pain, soc: Social Functioning.

Figure 1: Amount of patients showing comprehension problems with single items of the SF-36 dimensions (i.e. questions/comments). Items with a lowe score than 5 are not shown; $\mathrm{N}=86$.

\begin{tabular}{|c|c|c|c|c|c|c|c|c|c|c|}
\hline Scores & PF & RP & BP & GH & VT & SF & RE & MH & PCS & MCS \\
\hline M & 26.85 & 14.61 & 43.07 & 47.70 & 34.27 & 59.88 & 40.83 & 56.07 & 29.07 & 43.64 \\
\hline SD & 24.40 & 26.89 & 32.69 & 15.32 & 18.50 & 32.03 & 45.92 & 19.86 & 8.37 & 11.52 \\
\hline N & 86 & 81 & 86 & 86 & 85 & 86 & 80 & 85 & 79 & 79 \\
\hline
\end{tabular}

M: Mean Value; SD: Standard Deviation; N: Sample Size; PF: Physical Functioning; RP: Role-Physical; BP: Bodily Pain; GH: General Health; VT: Vitality; SF: Social Functioning; RE: Role-Emotional; MH: Mental Health; PCS: Physical Component Summary; MCS: Mental Component Summary

Table 2: Descriptive statistics for the dimensions and the summary scores of the SF-36. omitted one or more items. As not enough items were answered, the SF-36 summary scores could not be calculated for six participants from this group. $27.5 \%(11 / 40)$ of the patients who received strong assistance omitted one or more items (Figure 1). For one participant the summery score could not be calculated.

The EQ-5D-5L index values of nine rehabilitation patients could not be calculated due to missing data. All missing values were on item 3. Participants showed the most amount of comprehension problems for this item ( $\mathrm{N}=15$, Appendix 3 on the website). All participants were able to place a value on the EQ-5D-5L visual analogue scale.

\section{HRQoL questionnaire results}

The mean value of the SF-36 Role Physical dimension was the lowest $(M=14.61, N=81, S D=26.89)$, the one of the Social Functioning dimension the highest $(\mathrm{M}=59.88, \mathrm{~N}=86, \mathrm{SD}=32.03)$. All data in detail are depicted in Table 2. Floor and ceiling effects were considered present if more than $20 \%$ of the patients achieved the worst score $(0 / 100)$, the best score (100/100) respectively. The Role Physical dimension showed very pronounced floor effects $(70.4 \%$ of patients achieved $0 / 100)$ and the Role Emotional dimension showed both floor and ceiling effects (51.3\% or $33.8 \%$ achieved $0 / 100$ or $100 / 100)$. The EQ-5D-5L mean index value was $\mathrm{M}=0.60(\mathrm{~N}=77, \mathrm{SD}=0.27)$. The mean value of $\mathrm{EQ}-5 \mathrm{D}$ 5L VAS was $\mathrm{M}=59.24(\mathrm{~N}=86, \mathrm{SD}=20.68)$.

\section{Comparisons with representative samples}

Compared to the SF-36 German representative sample of the oldest age group ( $>70$ years, $\mathrm{N}=457)$ from 1998 [14] the mean scale scores of the geriatric rehabilitation patients were significantly lower in all dimensions $(\mathrm{p}<.0001)$ (Appendix 4 on the website). Comparisons with a representative sample for the EQ-5D-5L of German individuals aged 75 and over $(\mathrm{N}=247)$ Szende et al. [15] show that the mean index value of the current sample is significantly lower $(\mathrm{p}<.0001, \mathrm{M}=0.77, \mathrm{SD}=0.204)$. The results of the VAS of the EQ-5D-5L differ not significantly $(M=60.5$, $\mathrm{SD}=23.57$ ) from results of the here presented study.

\section{Correlation of the SF-36 with the EQ-5D-5L}

We carried out correlation analysis comparing the index values of the EQ-5D-5L with the physical (PCS) and mental summary scores (MCS) of the SF-36. A significant moderate relationship between the PCS and the EQ-5D-5L index value was found $(r=0.34, p<0.01)$. The MCS of the SF-36 did not correlate significantly with the index values of the EQ-5D-5L $(r=0.23, \mathrm{p}=0.052)$.

\section{Discussion}

\section{Feasibility and suitability of the SF-36 and EQ-5D-5L}

The present study confirms that both questionnaires can be completed by multimorbid geriatric rehabilitation inpatients in general. However, almost half of the participants needed strong assistance to complete the SF-36. Considering the limitations in human and time resources the suitability of the SF-36 for a routinely measurement of HRQoL, as recommended [4-6], is questionable for the in-patient geriatric rehabilitation setting. The additional time staff would need to spend for the assistance would be enormous (e.g. 267.1 hours/year for a medium-sized rehabilitation clinic with 1200 patients/year). The EQ-5D-5L can be handled quickly and without major complications. It seems therefore suitable for routinely use in an in-patient geriatric rehabilitation setting. The focus of the EQ-5D-5L, however, is on physical health. The use of the EQ-5D-5L would thus result in less consideration of mental health, as opposed to the use of the SF-36. The difference in the items relating to mental health becomes evident in the 
Citation: Grund S, Breitinger E, Fricke S, Alpers GW, Hundsdörfer W, et al. (2017) Health-Related Quality of Life Measurement Inpatient Geriatric Rehabilitation: A Comparison of the Feasibility and Suitability Between the SF-36 and EQ-5D-5L Questionnaires. J Gerontol Geriatr Res 6: 458. doi:10.4172/2167-7182.1000458

Page 3 of 4

lack of correlation between the mental health measures of the SF-36 with the EQ-5D-5L indices.

\section{Contents of the items}

In line with previous studies, it seems that elderly people omit items which seem to be not relevant for them [11]

[12]. This lead to a high amount of missing data, especially in the SF-36. The participants of the present study regarded questions of the dimensions Role Physical and Role Emotional about complex movements as inappropriate and were irritated by the addition of phrases like 'with daily activities, at work or at home'. To counteract these problems modification of the respective items should be explored, for example with the goal of another phrasing as has been already suggested [12] or a larger range of answer options. Müller et al. developed a SF-36 version suitable for hospitals [16]. They left out the critical item phrasing 'at work or at home'. The alteration resulted in a decreased number of missing values, when carrying out the survey with in-patients. However, mostly due to the fact that the questions themselves were not changed, this version has not become widely accepted in practice.

The questions of the EQ-5D-5L seem to be more suitable for geriatric rehabilitation in-patients. The answers are phrased in whole sentences which simplifies the understanding and hence selfcompletion. Nonetheless, item 3 which refers to 'usual activities (e.g. work, family or leisure activities)' irritated some participants. All nine missing EQ indices couldn't be calculated due to the missing value of item 3 .

\section{Floor and ceiling effects of the SF-36}

Floor and ceiling effects may arise due to the polar question form employed, which only allows for two answer options and was used for both the Role Physical and the Role Emotional dimension. The effects may also reflect physical limitations that necessitated rehabilitation in the first place. Data of indication-based rehabilitation clinics show similar results [17].

\section{Comparisons with representative samples}

Compared with representative samples the SF-36 and EQ-5D-5L scores reflect clearly the physical deficits which have led to geriatric rehabilitation. The very low ratings on the SF-36 physical dimensions and clearly higher values for the mental dimensions might be explained the 'satisfaction paradox' [18]. Moreover, there is good cause to hope for improvement in day-to-day functioning and for increased participation in social life [19] as patients are usually able to go back home after in-patient geriatric rehabilitation $[20,21]$. The fact that the EQ-VAS scores differ not significantly between the representative and rehabilitation sample may be also explained by the satisfaction paradox.

\section{Limitations}

Limitations of the present study are the observational character, the sample of only one institution as well as the sample size itself.

\section{Conclusion}

The results of this study confirm the general feasibility of the SF-36 and the EQ-5D-5L with indicated substantive modifications to improve efficiency and accuracy for measuring HRQoL in multimorbid geriatric rehabilitation in-patients. However, the items of the Role Physical, Role Emotional and Physical Functioning dimensions of the SF-36 and item 3 of the EQ-5D-5L need to be optimized for the geriatric rehabilitation setting. With regard to the limited time and human resources, the EQ$5 \mathrm{D}-5 \mathrm{~L}$ seems to be more suitable than the SF-36 for a routinely use in geriatric rehabilitation clinics. Due to the items of the EQ-5D-5L, mental health might be underrepresented compared to the SF36.

\section{Conflict of Interest}

All authors declare that they have no conflict of interest.

\section{Ethical Approval}

All procedures performed in studies involving human participants were in accordance with the ethical standards of the institutional and national research committee and with the 1964 Helsinki declaration and its later amendments or comparable ethical standards.

\section{Informed Consent}

Informed consent was obtained from all individual participants included in the study.

\section{References}

1. Gutenbrunner C (2007) Fundamentals of rehabilitation. In: Gutenbrunner C, Glaesener JJ (eds.), Rehabilitation, physical medicine and naturopathic treatment. Springer, Heidelberg, Germany. pp. 113-114.

2. Federal Association of Rehabilitation (2006). A tool for geriatric rehabilitation http://www.bar-frankfurt.de/fileadmin/dateiliste/publikationen/arbeitshilfen/ downloads/. Accessed 24 July 2016.

3. Hickey A, Barker M, McGee H, O'Boyle C (2005) Measuring health-related quality of life in older patient populations. Pharmaco Economics 23: 971-993.

4. German Society of Orthopaedics, Orthopaedic Surgery and the Federa Association of Doctors for Orthopaedic (DGOOC) (2013) Rehabilitation after major amputies of the lower extremities (proximal of the foot). http://www.awmf org/uploads/tx_szleitlinien/033-044I_S2k_Rehabilitation_Majoramputationuntere_Extremitaet_2013-09.pdf. Accessed 24 July 2016

5. Diener HC (2012) Guidelines for diagnostic and therapy in neurology (5th edn). Thieme, Stuttgart, Germany.

6. Fischer J, Schnabel M, Sitter H (2007) Rehabilitation of patients with chronic obstructive pulmonary disease (COPD). S2 guideline of the German society for pneumology and respiratory medicine and the German society for rehabilitation science (RGRW). Pneumologie.

7. PubMed. SF 36 - Results by year. National Center of Biotechnology Information (NCBI), U.S National Library of Medicine. http://www.ncbi.nlm.nih.gov/ pubmed/?term=SF+36. Accessed 28 July 2016.

8. PubMed. EQ-5D - Results by year. National Center of Biotechnology Information (NCBI), U.S National Library of Medicine. http://www.ncbi.nlm.nih gov/pubmed/?term=EQ+5D. Accessed 28 July 2016.

9. Bullinger M (2000) Recording of health-related quality of life using SF-36-Health Survey. Bundesgesundheitsblatt - Gesundheitsforschung-Gesundheitsschutz, Germany.

10. Janssen MF, Pickard AS, Golicki D, Gudex C, Niewada M, et al. (2013) Measurement properties of the EQ-5D-5L compared to the EQ-5D-3L across eight patient groups: a multi-country study. Qual Life Res 22: 1717-1727.

11. Brazier JE, Walters SJ, Nicholl JP, Kohler B (1996). Using the SF-36 and Euroqol on an elderly population. Qual Life Res 5: 195-204.

12. Hayes V, Morris J, Wolfe C, Morgan M (1995) The SF-36 health survey questionnaire: Is it suitable for use with older adults? Age and Ageing 24: 120-125.

13. EuroQol Research Foundation (2016). EQ-5D-5L Value Sets. http://www. euroqol.org/about-eq-5d/valuation-of-eq-5d/eq-5d-5l-value-sets. html. Accessed 28 July 2016.

14. Morfeld M, Kirchberger I, Bullinger M (2011) SF-36: Health Survey (2nd edn). Göttingen: Hogrefe Verlag GmbH \& Co. KG, Germany.

15. Szende A, Janssen B, Cabases J (2014) Self-reported population health: an international perspective based on EQ-5D. Springer, Heidelberg, Germany.

16. Mueller H, Franke A, Schuck P, Resch KL (2000)A hospital suited version of the German SF-36 and its psychometric comparison with the original questionnaire. Soz Praventivmed.

17. Kupatz P, Thielker K (2014) Comparative study of two instruments to measure 
Citation: Grund S, Breitinger E, Fricke S, Alpers GW, Hundsdörfer W, et al. (2017) Health-Related Quality of Life Measurement Inpatient Geriatric Rehabilitation: A Comparison of the Feasibility and Suitability Between the SF-36 and EQ-5D-5L Questionnaires. J Gerontol Geriatr Res 6: 458. doi:10.4172/2167-7182.1000458

Page 4 of 4

healt-related quality of life in orthopaedic, rheumatological, psychosomatic and neurological rehabilitation. Report April 2013. Verein zur Förderung der Rehabilitationsforschung in Hamburg, Mecklenburg-Vorpommern und Schleswig-Holstein e.V.

18. Herschbach $P(2002)$ The 'satisfaction paradox' on the research of quality of life. Psychother Psych Med 52: 141-150.

19. World Health Organisation (2015). World Health Organisation - International
Classification of function (ICF). http://www.who.int/classifications/icf/en/. Accessed 9 September 2016

20. Jamour M, Marburger C, Runge M, Sieber CC, Tümena T (2014) Effectiveness of geriatric rehabilitation in the oldest old. Z Gerontol Geriatr 47: 389-396.

21. Grund S, Mettlach M, Kieser M, Rath K, Schäfer HG (2015) Outcomes of multimorbid vascular surgery patients with and without major amputation in a German geriatric rehabilitation clinic. Z Gerontol Geriatr. 